\title{
Spindle Cell Carcinoma of the Head and Neck: A Clinicopathological and Immunohistochemical Study of 40 Cases
}

\author{
Anupam Sarma', Rajjyoti Das ${ }^{2}$, J. D. Sharma', A. C. Kataki ${ }^{3}$ \\ ${ }^{1}$ Department of Pathology, Dr. B. Borooah Cancer Institute, Guwahati, India; ${ }^{2}$ Department of Head \& Neck Oncology, Dr. B. \\ Borooah Cancer Institute, Guwahati, India; ${ }^{3}$ Department of Gynecologic Oncology \& Director, Dr. B. Borooah Cancer Institute, \\ Guwahati, India. \\ Email: dranupamsarma@gmail.com
}

Received September $24^{\text {th }}, 2012$; revised October $26^{\text {th }}, 2012$; accepted November $6^{\text {th }}, 2012$

\begin{abstract}
Spindle cell carcinoma of head and neck, a subtype of squamous cell carcinoma is a unique and rare neoplasm. It has a more aggressive behavior as compared to classical squomous cell carcinoma warranting surgical interventions with wider surgical margins. Immunohistochemistry along with routine histopathology is essential in establishing the diagnosis of spindle cell carcinoma. We at Dr. B. Borooah Cancer Institute, Guwahati, a regional institute for treatment and research, hereby report 40 cases of such lesion with clinicopathological and immunohistochemical study. Out of total 40 cases included in the study group most of the cases were in the age group of 40 to 60 years. Commonest site of presentation was nasopharynx and buccal mucosa. 14 cases of the oral cavity (buccal mucosa, alveolus, oral tongue and hard palate) were treated with surgery. All the cases with disease of the larynx and hypopharynx were treated with radiotherapy and cases involving the nasopharynx received radiotherapy and chemotherapy. In the surgery group recurrence rate was found to be $71.4 \%$ and metastasis rate was $21.4 \%$. Biopsy specimens were subjected to histopathological examination followed by immunohistochemistry. Immunohistochemical analysis show concurrent presence of malignant epithelial and sarcomatoid spindle cell components by co-expression of cytokeratin (CK) and vimentin to various degrees.
\end{abstract}

Keywords: Spindle Cell Carcinoma (SpCC); Squamous Cell Carcinoma (SCC); Immunohistochemistry (IHC)

\section{Introduction}

Spindle cell carcinoma (SpCC) designates a rare variant growth pattern of squamous cell carcinoma (SCC) in which the spindle epithelial cell resembles a sarcoma on histological examination. It is also known as pseudosarcoma, carcinosarcoma, sarcomatoid squamous cell carcinoma or polypoid squamous cell carcinoma. It is usually present as a large polypoid, pedunculated neoplasm, protruding from the mucosal surface with ulceration. Spindle cell carcinoma is most commonly seen in the head and neck region. Most cases in the head and neck arise in the oral cavity, larynx, tonsil and pharynx. However, it may occur in other sites of body also. Symptoms vary according to the site. The mean age of appearance for SpCC is the sixth decades of life and it has a male predominance. The predisposing factors are the same as SCC, including tobacco use, alcohol abuse, poor oral hygiene and previous irradiation to the site in which the tumor arise. It poses a diagnostic dilemma when con- fronted with malignant tumor with spindle cell morphology in the head and neck region, keeping in mind that it could be a variant of conventional SCC for therapeutic consideration.

\section{Methods}

A retrospective study from 2006 to 2012, of 7500 cases of SCC of head and neck region at Dr. B. Borooah Cancer Institute, Guwahati, a regional institute for treatment and research were selected. Inclusion and exclusion of cases for the study was carried out by reviewing the biopsy specimens histologically. In histopathological examination definitive SCC were excluded and cases which were found to be an unusual form of poorly differentiated SCC consisting of elongated spindle cells of various proportions that resemble a sarcoma were included in the study. Those cases were subsequently subjected to immunohistochemistry with cytokeratin (CK) and vimentin. In few selected cases some other markers like S-100 
were also performed. All possible clinical data were evaluated. This study is compliant with the requirements of local research ethics committee.

\section{Results}

Most of the cases were in the age group of 40 to 60 years (Table 1). Youngest case was a 24 years old male and the oldest was an 80 years old male. It was found to be predominant amongst males with a male to female ratio of 34:6. Commonest site of presentation was nasopharynx and buccal mucosa (Table 2).

On external examination, all the patients with lesion in the lower alveolus present with diffuse swelling in the region of the mandible (Figure 1). Patient with lesion in the base of tongue (BOT) and pyriform sinus (PFS) mostly present with difficulty in swallowing. Patient with the growth in the nasopharynx mostly present with the nasal bleeding and one or both sided nasal obstruction. Intraoral examination most of the lesions are either in the lower alveolus or hard palate and found mostly irregular surface and tender on palpation. All the patient requiring

Table 1. Age groups of presentation of spindle cell carcinoma of head and neck.

\begin{tabular}{cc}
\hline Age & No. of cases \\
\hline$<40$ & 6 \\
$40-60$ & 20 \\
$>60$ & 14 \\
\hline
\end{tabular}

Table 2. Sites of presentation of spindle cell carcinoma of head and neck.

\begin{tabular}{ccc}
\hline & SITE & No. of cases \\
\hline 1 & Nasopharynx & 8 \\
2 & Buccal mucosa & 6 \\
3 & Alveolus & 4 \\
4 & Base of tongue & 4 \\
5 & Pyriform sinus & 4 \\
6 & Palate & 3 \\
7 & Larynx & 3 \\
8 & Tonsil & 3 \\
9 & Maxilla & 1 \\
10 & Valecula & 1 \\
11 & Posterior pharyngeal wall & 1 \\
12 & Aeryepiglottic fold & 1 \\
13 & Oral tongue & 1 \\
\hline
\end{tabular}

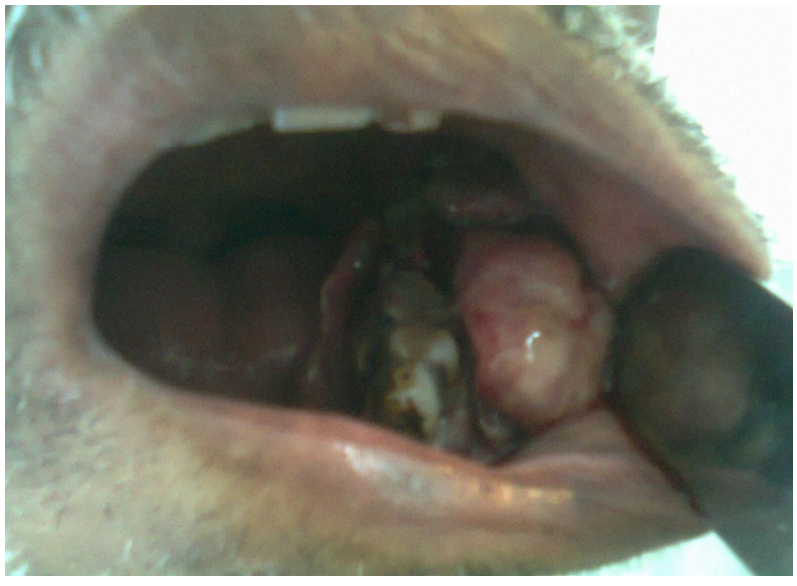

Figure 1. Intraoral examination shows a growth in the lower alveolus involving lower buccal sulcus and mucosa.

surgery is put for routine haematological and radiological examination including OPG and CT scan.

Out of total 40 cases included in the study group, 14 cases of the oral cavity (buccal mucosa, alveolus, oral tongue and hard palate) were treated with surgery. All the cases with disease of the larynx and hypopharynx were treated with radiotherapy and cases involving the nasopharynx received radiotherapy and chemotherapy. In the surgery group 10 patients (recurrence rate $71.4 \%$ ) developed local recurrence out of which 6 patients had recurrence in the primary site, 4 patients in the lymph nodes and 3 patients had distant metastasis (metastasis rate $21.4 \%$ ) and died subsequently, out of the 14 cases 10 cases received salvage surgery. Patient with disease in the oropharynx, nasopharynx and larynx received radical radiotherapy up to dose of 66 - $70 \mathrm{~Gy}$ in fractions for a period of 5 to 7 weeks. In chemotherapy mostly used multi drug regime with Cisplatin, Carboplatin and 5Fluorourasil.

\section{Histopathology and Immunohistochemistry}

Some biopsy specimen revealed only the monophasic sarcomatoid component and other specimen revealed biphasic features. The squamous component is found mostly non keratinizing (poorly differentiated) and the spindle cell component showed elongated cells, hyperchromatic nuclei with many mitosis (Figure 2). The chief differential diagnoses of spindle cell carcinoma are true superficial sarcomas and benign but atypical reactive angiofibroblastic or myofibroblastic proliferations involving the mucosa. Because sarcomas and reactive pseudosarcomatous proliferations arising from the submucosa in the head and neck region are exceedingly rare, the assumption that a polypoid mucosal malignant spindle cell proliferation is a spindle cell carcinoma is reasonable. 


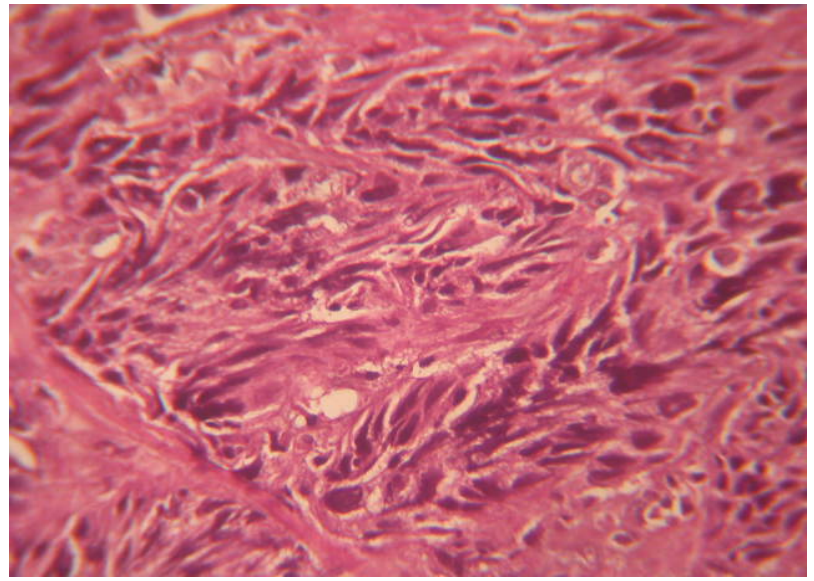

Figure 2. Photomicrograph showing sarcoma like spindle shaped tumor cells with pleomorphism and hyperchromatism of nuclei and mitosis (H\&E, 400×).

Immunohistochemical (IHC) analysis of 40 cases of SpCC of the head and neck region were carried out to establish the usefulness of selected IHC markers in distinguishing SpCC from other mucosal spindle-cell neoplasms. IHC analysis using polymer HRP detection system and DAB chromogen show concurrent presence of malignant epithelial and sarcomatoid spindle cell components by co-expression of cytokeratin (CK) and vimentin to various degrees (Figures 3 and $\mathbf{4}$ ). All the SpCC where S-100 protein was carried out shown negative immunoreactivity.

Cytokeratins are a family of water-insoluble proteins forming a major part of the cytoskeleton of epithelial cells and thus forms an important aid in the classification of epithelial neoplasm. Vimentin on the other hand is the major intermediate filament in a variety of mesenchymal cells. S-100 protein derives its name from the fact that it is soluble in saturated ammonium sulphate solution. By IHC analysis spindle cell sarcoma, melanoma and malignant myoepithelioma can be ruled out.

\section{Discussion}

The different variant of SCC of the head and neck region comprising of around 15\% of SCC are verrucous, exophytic or papillary, spindle-cell (sarcomatoid), basaloid and adenosquamous carcinoma. A different histopathologic and clinical feature also leads to different diagnostic considerations as well as different management approach. Spindle cell carcinoma is an uncommon tumour accounting for 3\% of SCC [1].

In the past SpCC has been thought to be a collision tumour between carcinoma and sarcoma. Virchow was the first person to introduce the term carcinosarcoma for this group of lesions. Kettle E. H., in the beginning of 20th century described that many authors were of the opinion

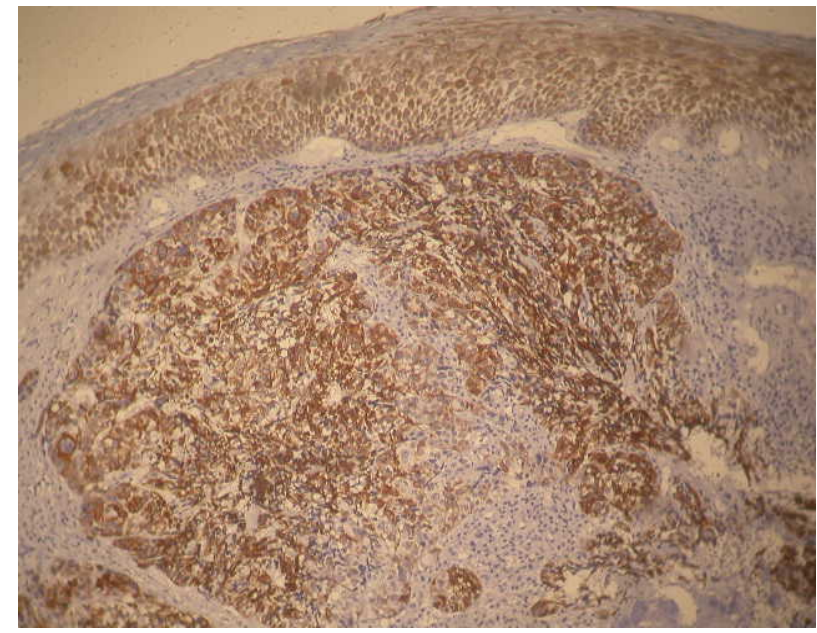

Figure 3. Immunohistochemical staining showing tumor cells are positive for $\mathrm{CK}$. Epithelium is also showing positive staining (Pan Cytokeratin, 100×).

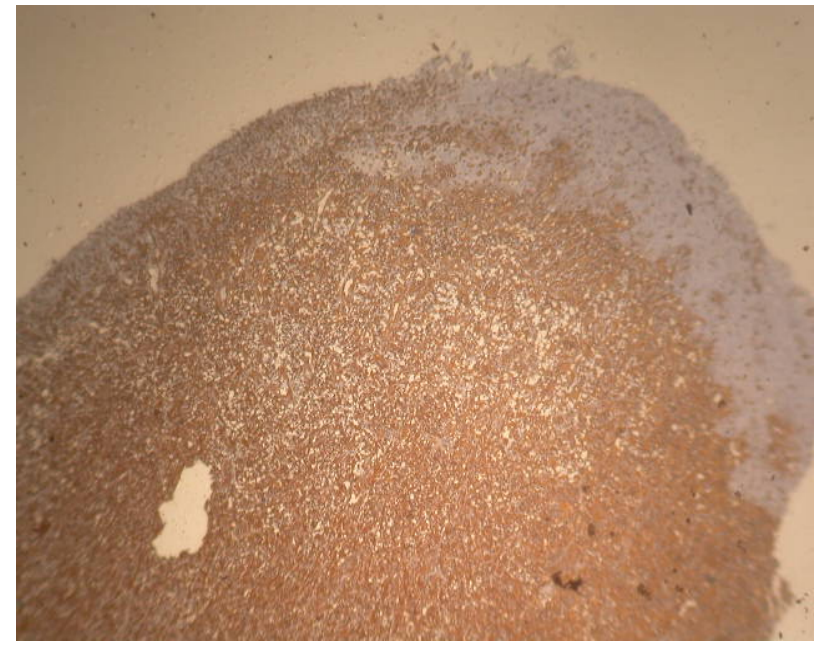

Figure 4. Immunohistochemical staining showing tumor cells are positive for vimentin. Epithelium showing negative staining (Vimentin, 100×).

that the spindle cell component is actually epithelial cells being transformed into mesenchymal cells [2].

Numerous hypotheses regarding the histogenesis of SpCC have been proposed. Three dominant pathogenetic theories have been proposed: the tumor 1) represents a "collision tumour" (carcinosarcoma), 2) is a squamous cell carcinoma with an atypical reactive stroma (pseudosarcoma), or 3) is of epithelial origin, with "de-differentiation" or transformation to a spindle cell morphology (sarcomatoid carcinoma) [3]. Recently, the third hypothesis has been supported by following evidences: their occurrence in the exact sites that normally have squamous epithelium and a preponderance of carcinomas rather than sarcomas; a superficial location; a polypoid appearance; the direct continuity and smooth transition of 
the spindle cells with areas of squamous epithelium; immunoreactivity with epithelial antigens; a dual expression of epithelial and mesenchymal differentiation with double labeling techniques in some neoplastic spindle cells; and the presence of epithelial only, sarcomatous only, or a duality of expression in metastatic deposits from laryngeal sarcomatoid carcinoma [4].

The mean age of diagnosis of Spindle cell carcinoma is 57 years. However it can be diagnosed in younger age group and very old age group (range 29 - $93 \mathrm{yr}$ ). As far the gender predilection is concerned most authors reported that there is equal frequency in both males and females. Some studies have reported a male predilection [5]. Our cases presented in the correct decade and mean age was 52.8 years with male predilection.

Site predilection for SpCC are the lower lip, tongue and alveolar ridge or gingival although most tumours in head and neck region occur in the larynx [6]. Growth configuration is often exophytic polypoid, but sessile, nodular or endophytic configuration has also been described. The lesion usually has an extensive surface ulceration with friable, fibrinoid necrosis of variable thickness or shaggy exudates. Radiation, trauma, tobacco use or alcohol consumption seemed to play a role in etiological factors [7].

Spindle cell carcinoma can demonstrate varied histopathological appearance from case to case or within different areas of the same tumour tissue. The cells may appear like epithelial cells or may appear as atypical mesenchymal cells to add to the confusion. A histopathologist using a light microscope may find it extremely difficult to assess the case as it may appear as a carcinoma as well as a sarcoma. The tumour generally consists of fascicles of anaplastic spindle cells with considerable number of mitotic figures [8].

Differential diagnosis includes a number of benign and malignant tumours, such as fibromatosis, nodular fasciitis, reactive epithelial proliferations, squamous cell carcinoma, fibrosarcoma, malignant fibroushistocytoma, leiomyosarcoma, rhabdomyosarcoma, malignant peripheral nerve sheath tumour, mesenchymal chondrosarma and malignant melanoma [4].

The treatment in case of SpCC has been debated. Many are in the opinion that wide excision which includes radical dissection along with metastasis is adequate. Some authors are of the opinion that surgery alone is not sufficient in that radiotherapy is mandatory along with surgery. Radiotherapy is considered an acceptable alternative for inoperable patients. Radiotherapy also play important role in case with surgical margin positive or extensive nodal disease. Unfortunately $30 \%$ of all oral cases, most cases were ending fatally within one year. This is similar in connection with the high grade SCC.
Presence of metastasis signals a poor prognosis as per study conducted by Ellis and Corio and this applicable in cases of oral cavity [9]. In a study out of total 15 patients of SpCC of oral cavity and oropharynx receiving surgery, recurrence rate was $73.3 \%$ and metastasis rate $33.3 \%$ was reported [10]. In our series the overall recurrence rate was found to be $71.4 \%$ and metastasis rate of $21.4 \%$.

Immunohistochemical characterization of tumour cells using antibodies to keratin, vimentin, and S-100 protein is very helpful in differentiating SpCC from true spindle cell sarcoma, melanoma and malignant myoepithelioma. In the IHC, it is important to remember that SpCC should not be ruled out of the differential diagnosis by a positive reaction for vimentin in sarcomatoid tumour cells. Absence of staining for keratin in the sarcomatoid tumour cells does not always exclude SpCC because some SpCC show immunoreactivity of keratin in their sarcomatoid components only with some anti-keratin antibodies. Different kinds of anti-keratin antibodies should be applied in the differential diagnosis of SpCC [11]. IHC analysis of our cases show co-expression of cytokeratin (CK) and vimentin to various degrees. In our study Pan cytokeratin has been used which includes wide range of cytokeratin antibodies.

\section{Conclusion}

SpCC is a neoplasm of epithelial origin and considered to be a variant of SCC. It mimics other connective tissue sarcomas and spindle cell malignancies under light microscopy. Immunohistochemistry is helpful to know the histogenesis and nature of SpCC. Prognosis of the disease always poor and distant metastasis is always more then the conventional SCC.

\section{REFERENCES}

[1] L. D. R. Thompson, "Squamous Cell Carcinoma Variants of the Head and Neck," Current Diagnostic Pathology, Vol. 9, No. 6, 2003, pp. 384-396. doi:10.1016/S0968-6053(03)00069-3

[2] E. H. Kettle, "On Polymorphism of Malignant Epithelial Cell," Proceedings of the Royal Society of Medicine, Vol. 12, 1919, pp. 1-32.

[3] S. F. Huang, I. H. Chen, C. T. Liao, T. M. Chen and K. F. Lee, "Sarcomatoid Carcinoma of the Parotid Gland with Apparent Metastasis of Epidermoid Elements to Cervical Lymph Nodes,” Acta Oto-Laryngologica, Vol. 126, No. 6, 2006, pp. 667-671. doi:10.1080/00016480500469560

[4] L. D. Thompson, J. A. Wieneke, M. Miettinen and D. K. Heffner, "Spindle Cell (Sarcomatoid) Carcinomas of the Larynx: A Clinicopathologic Study of 187 Cases,” American Journal of Surgical Pathology, Vol. 26, No. 2, 002, pp. 153-170.

[5] G. Randall, W. A. Alonso and J. H. Ogura, "Spindle Cell 
Carcinoma (Pseudosarcoma) of the Larynx," Archives of Otolaryngology, Vol. 101, No. 1, 1975, pp. 63-66. doi:10.1001/archotol.1975.00780300067018

[6] C. Rizzardi, C. Frezzini, M. Maglione, G. Tirelli and M. Melato, "A Look at the Biology of Spindle Cell Squamous Carcinoma of the Oral Cavity: Report of a Case," Journal of Oral and Maxillofacial Surgery, Vol. 61, No. 2, 2003, pp. 264-268. doi:10.1053/joms.2003.50025

[7] S. B. Minami, S. Shinden and T. Yamashita, "Spindle Cell Carcinoma of the Palatine Tonsil: Report of a Diagnostic Pitfall and Literature Review," American Journal of Otolaryngology, Vol. 29, No. 2, 2008, pp. 123-125. doi:10.1016/j.amjoto.2007.02.006

[8] B. W. Neville, D. D. Damm, C. M. Allen and J. E. Bouquot, "Oral and Maxillofacial Pathology: Saunders Elsivier,” 3rd Edition, Elsevier, Toronto, 2005, pp. 369-370.
[9] G. L. Ellis and R. L. Corio, "Spindle Cell Carcinoma of the Oral Cavity: A Clinicopathologic Assessment of Fifty Nine Cases," Oral Surgery, Oral Medicine, Oral Pathology and Oral Radiology, Vol. 50. No. 6, 1980, pp. 523533.

[10] H.-H. Su, S.-T. Chu, Y.-Y. Hou, K.-P. Chang and C.-J. Chen, "Spindle Cell Carcinoma of the Oral Cavity and Oropharynx: Factors Affecting Outcome,” Journal of the Chinese Medical Association, Vol. 69, No. 10, 2006, pp. 478-483. doi:10.1016/S1726-4901(09)70312-0

[11] T. Takata, H. Ito and I. Ogawa, "Spindle Cell Squamous Carcinoma of the Oral Region. An Immunohistochemical and Ultrastructural Study on Histogenesis and Differential Diagnosis with Clinicopatholological Analysis of Six Cases," Virchows Archiv A, Pathological Anatomy and Histology, Vol. 419, 1991, pp. 177-182. doi:10.1007/BF01626345 